\title{
Anabases
}

ANABASES Traditions et réceptions de l'Antiquité

$17 \mid 2013$

Varia

\section{Des échos virgiliens dans un Voyage Extraordinaire de Jules Verne, Le Château des Carpathes (1892)}

Lionel Dupuy

\section{(2) OpenEdition}

\section{Journals}

Édition électronique

URL : http://journals.openedition.org/anabases/4088

DOI : 10.4000/anabases.4088

ISSN : 2256-9421

Éditeur

E.R.A.S.M.E.

\section{Édition imprimée}

Date de publication : 1 mars 2013

Pagination : 27-39

ISSN : 1774-4296

\section{Référence électronique}

Lionel Dupuy, «Des échos virgiliens dans un Voyage Extraordinaire de Jules Verne, Le Château des

Carpathes (1892) », Anabases [En ligne], 17 | 2013, mis en ligne le 01 avril 2016, consulté le 08 janvier 2020. URL : http://journals.openedition.org/anabases/4088 ; DOI : 10.4000/anabases.4088

(c) Anabases 
Anabases 17 (2013), p. 27-39.

\section{Des échos virgiliens \\ dans un Voyage Extraordinaire de Jules Verne, Le Château des Carpathes (1892)}

Lionel DupuY

En 1892, Jules Verne (1828-1905) publie Le Château des Carpathes, un roman où l'auteur met en scène au début de l'aventure un modeste berger - personnage a priori anecdotique dans l'histoire. Or, la présentation de ce pâtre se fait par le truchement de la transformation d'une citation latine virgilienne que Victor Hugo utilise comme titre du chapitre célèbre de Notre-Dame de Paris où il introduit le personnage de Quasimodo : Immanis pecoris custos, immanior ipse ${ }^{1}$. Or, comme le souligne Yves Touchefeu en 2005, dans un texte qui s'intéresse également aux occurrences de cette formule chez Jules Verne ${ }^{2}$, cette citation latine transformée apparait ici pour la quatrième et dernière fois dans l'œuvre du romancier français.

Nous souhaiterions dans cet article compléter et approfondir dans un premier temps les différents points abordés par Yves Touchefeu (I et II) ${ }^{3}$, afin de montrer

1 Victor Hugo, Notre-Dame de Paris. Les Travailleurs de la mer, chapitre III, Livre IVe, éd. Y. Gohin et J. Seebacher, Paris, Gallimard, Bibliothèque de la Pléiade, 1975, p. 147.

2 Y. Touchefeu, "Jules Verne latiniste : présences du latin et de la culture classique dans les Voyages Extraordinaires", article de 2005, non publié, en ligne : http://aplg.pagespersoorange.fr/Jules\%20Verne\%20latiniste.doc (visité le 10 juillet 2012).

3 Dans son analyse, Y. Touchefeu développe plus spécifiquement le rapprochement avec Victor Hugo, la référence au Protée des Géorgiques de Virgile, ou encore les maladresses de Jules Verne dans ses traductions du latin au français et dans son maniement du latin lui-même. Vingt ans auparavant, Simone Vierne faisait déjà référence à cette déformation, qui apparaît dès le Voyage au centre de la Terre, dans son ouvrage Jules Verne. Une vie, une auvre, une époque, Paris, Balland, 1986, p. 154-155. En 2009, Monique Crampon consa- 
ensuite (III et IV) plus spécifiquement comment ces différents éléments participent aussi - et avant tout - à la construction de la géographie et de l'imaginaire géographique de ce roman emblématique de la série des Voyages Extraordinaires qu'est Le Château des Carpathes ${ }^{4}$. Dans cette perspective, l'hypothèse que nous formulons est que les références internes (intratexte) et externes (intertexte) permettent à Jules Verne d'établir un pont entre l'œuvre de Virgile, celle de Victor Hugo et la sienne, tout en faisant référence également à ses propres romans, dont soixante-deux composent le corpus des Voyages Extraordinaires (V.E. $)^{5}$. Mais la reprise de ce vers issu originellement des Bucoliques de Virgile lui sert aussi à faire évoluer ses héros dans un imaginaire géographique favorisé par la narration d'un récit qui se déroule aux marges de l'Europe, en Roumanie, et plus particulièrement en Transylvanie, dans les Carpates ${ }^{6}$.

Dans cette aventure, le berger Frik fait observer aux habitants du modeste village de Werst que de la fumée s'échappe du château qui surplombe la petite localité. Or, ce dernier est considéré comme abandonné depuis longtemps, ce qui ne manque pas d'inquiéter les habitants très enclins à envisager avant tout cet événement comme surnaturel et fantastique ${ }^{7}$. Ce récit est typique des V.E. : nous proposons ainsi de les lire comme des romans géographiques qui sont articulés autour du passage entre une géographie scientifique, campant le récit dans un réel confirmé, et une géographie plus fictive, ancrée dans l'imaginaire et le fantastique.

cre deux pages (qui rejoignent les propos d'Y. Touchefeu) à cette déformation du vers de Virgile dans l'œuvre de Jules Verne, dans un texte qui s'intéresse aux multiples avatars de cette citation latine dans la littérature classique et aux mutations qui affectent le champ lexical de la Beauté, avec l'avènement du romantisme. Voir M. CrAmpon, «Variations sur formosus, de Virgile à Jules Verne ", in Cl. BRunET (dir.), Des formes et des mots chez les Anciens : Mélanges offerts à Danièle Conso, Besançon, Presses Universitaires de FrancheComté, 2009, p. 51-62.

4 Nous nous permettons de renvoyer à notre thèse Géographie et imaginaire géographique dans les Voyages Extraordinaires de Jules Verne: Le Superbe Orénoque (1898), thèse de doctorat en géographie, dir. J.-Y. PuYo et V. Berdoulay, université de Pau et des Pays de l'Adour, 2009, en ligne : http://pagesperso-orange.fr/jules-verne/These_Lionel_Dupuy. pdf. Nous montrons dans notre thèse en quoi les Voyages Extraordinaires de Jules Verne sont avant tout des romans géographiques, appellation que l'auteur revendiquait déjà à son époque.

5 Concernant l'influence de Victor Hugo sur Jules Verne, voir L. DupuY, Géographie et imaginaire géographique, p. 65 sq.

6 Jules Verne écrit "Carpathes" tel que le nom était orthographié à son époque.

$7 \quad$ L'incipit du roman prend pourtant le contre-pied de cette interprétation a priori : "Cette histoire n'est pas fantastique, elle n'est que romanesque. Faut-il en conclure qu'elle ne soit pas vraie, étant donné son invraisemblance ? Ce serait une erreur " (chap. I, p. 7). J. Verne, Le Château des Carpathes, Paris, Le Livre de Poche, 2001. 


\section{De Virgile à Victor Hugo : aux origines du vers}

Au XIX siècle l'œuvre de Virgile joue un rôle important dans l'enseignement des humanités. Nous savons ainsi que Victor Hugo a fréquenté de près Virgile dans sa formation, ce dont témoignent ses cahiers de vers d'adolescent où figurent les traductions qu'il a réalisées de certaines Bucoliques. Le passage qui nous intéresse est situé aux vers 43 et 44 de la Bucolique V et sert d'épitaphe pour le tombeau de Daphnis : Daphnis ego in siluis hinc usque ad sidera notus, / formosi pecoris custos, formosior ipse ${ }^{8}$. Virgile recourt ici à un personnage déjà présent dans les Idylles de Théocrite : il s'agit du fils d'Hermès et d'une nymphe; il apparaît surtout comme le fondateur mythique du genre pastoral ${ }^{9}$.

Dans Notre-Dame de Paris (1831), Victor Hugo reprend et transforme le vers de Virgile afin d'en faire le titre du chapitre III du livre IV : Immanis pecoris custos, immanior ipse ${ }^{10}$. C'est dans ce chapitre précisément que l'auteur décrit Quasimodo, gardien des cloches de la cathédrale. Il y évoque particulièrement sa monstruosité, sa laideur, son caractère sauvage : "Il était méchant en effet, parce qu'il était sauvage ; il était sauvage, parce qu'il était laid. Il y avait une logique dans sa nature comme dans la nôtre ${ }^{11}$. "Victor Hugo transforme ici l'imaginaire du monde bucolique en une description grotesque et monstrueuse de son célèbre personnage. Par la transformation du positif en négatif, par cette hyperbole inversée, le romancier fait de Quasimodo un être à part, un monstre reclus dans un édifice religieux hautement symbolique, mais abîmé par le temps. Nous verrons notamment par la suite que les romans de Jules Verne, dont certains composent le corpus des V.E., participent aussi de cette logique qui consiste à intégrer de l'imaginaire, du fantastique, dans une aventure a priori ordinaire.

Dans l'œuvre de Virgile, il existe également un gardien monstrueux, difforme. Il s'agit de Protée qui surveille les troupeaux monstrueux de Neptune :

8 " Je suis Daphnis, habitant des forêts connu d'ici-bas jusqu'aux astres, gardien d'un beau troupeau, plus beau encore moi-même. ". Traduction tirée de la Revue de la Bibliothèque nationale, 31-34 (1989), p. 12.

9 R. Colemant, Vergil, Eclogues, Cambridge, Cambridge University Press, 1977, p. 1.

10 Comme le précise l'éditeur de Notre-Dame de Paris de la Pléiade, J. SeEbacher, Hugo a traduit lui-même cette formule, en marge d'un manuscrit d'Hernani : "Gardien d'un troupeau monstre, et plus monstre lui-même ». L'éditeur commente ainsi l'inversion réalisée par rapport à Virgile : « le monstrueux, qui semble inverser la beauté, la conserve dans son renversement " (Victor HugO, Notre-Dame de Paris. Les Travailleurs de la mer, p. 1141-1142). Remarquons au passage la mise en valeur du terme " monstre " par un écart avec l'adjectif attendu, "monstrueux ", qui montre justement que le terme n'est pas choisi au hasard par Hugo.

11 V. Hugo, Notre-Dame de Paris, chapitre III, Livre IVe, p. 150-151. 
" Est in Carpathio Neptuni gurgite vates,

Caruleus Proteus, magnum qui piscibus aquor

Et juncto bipedum curru metitur equorum ${ }^{12}$."

Il faut d'ailleurs souligner que nous retrouvons la référence latine à Protée dans un autre roman de Jules Verne, où l'intertextualité hugolienne est fortement marquée, Vingt mille lieues sous les Mers (1869-1870) :

"Aussi, je n'eus connaissance de l'île de Carpathos, l'une des Sporades, que par ce vers de Virgile que le capitaine Nemo me cita, en posant son doigt sur un point du planisphère :

Est in Carpathio Neptuni gurgite vates

Caruleus Proteus...

C'était, en effet, l'antique séjour de Protée, le vieux pasteur des troupeaux de Neptune, maintenant l'île de Scarpanto, située entre Rhodes et la Crète. Je n'en vis que les soubassements granitiques à travers la vitre du salon ${ }^{13}$."

Soulignons au passage que le nom de l'île (Carpathos) rappelle bien évidemment cette région de Roumanie où se déroule le récit que nous étudions ici. Précisons enfin pour terminer que le chapitre II du livre XI de Notre-Dame de Paris s'intitule ainsi : "La creatura bella bianco vestita (Dante). " Comment ne pas reconnaître ici une préfiguration du personnage de la belle Stilla (Le Château des Carpathes), qui, alors qu'elle est morte, apparaît pourtant de blanc vêtu aux imprudents visiteurs qui s'approchent du château?

\section{De Victor Hugo à Jules Verne : des références externes aux références internes}

Jules Verne semble affectionner particulièrement la transformation que Victor Hugo a faite du vers de Virgile. Nous la retrouvons à quatre reprises dans ses textes. Deux fois dans des récits hors des $V . E$., et deux fois dans des romans appartenant aux V.E. :

Paris au $X X^{\mathrm{e}}$ siècle, 1863, première publication en 1994 (hors V.E.),

Voyage au centre de la Terre, 1867 (V.E.),

Une Ville idéale : Amiens en l'an 2000, 1875 (hors V.E.),

12 «Il est au gouffre de Carpathos un devin de Neptune, Protée au corps d'azur, qui parcourt la grande plaine des mers sur un char attelé de coursiers à deux pieds, moitié poissons et moitié chevaux " (Virgile, Les Bucoliques et Géorgiques, trad. M. RAT, Paris, Classiques Garnier, 1932).

13 J. Verne, Vingt mille lieues sous les Mers, Paris, Gallimard, Le Livre de Poche, 1989, chap. VI, $2^{\mathrm{e}}$ partie, p. 368-368. 


\section{Le Château des Carpathes, 1892 (V.E.) ${ }^{14}$.}

Dans les deux textes hors du corpus des V.E., Jules Verne recourt à l'anticipation et parle respectivement de Paris et d'Amiens dans le futur, un siècle plus tard. Dans le premier texte, un récit initialement refusé par Hetzel, l'éditeur de Verne, le romancier imagine la vie à Paris en 1960, une vie où la culture classique a disparu au profit de la science et de la technique. En réalité, sous couvert d'anticipation, Jules Verne rédige une satire sociale et politique qui dénonce certains grands projets d'urbanisme et d'aménagement parisiens du Second Empire (1852-1870). Le déplacement du récit dans le temps permet au romancier de critiquer ce qu'il observe de son vivant. Mais si Victor Hugo, dans Notre-Dame de Paris, opère un déplacement de son récit vers le passé, Jules Verne opère quant à lui un déplacement vers le futur. Dans les deux cas, c'est bien la variable du temps, dans le chronotope romanesque, qui est mobilisée, assurant dès lors une mise à distance qui permet aux romanciers de porter un regard critique sur leur époque.

Le texte de Paris au $X X^{e}$ siècle est certes maladroit, incertain, très acide, mais il demeure particulièrement intéressant pour la recherche vernienne. En effet, l'inquiétude qui a toujours animé Jules Verne, mais qui a été contenue efficacement par son éditeur, transparaît ici très nettement ${ }^{15}$. Nous assistons dans le chapitre XI à la discussion suivante, où l'on se désole de ces rares élèves, qui bien qu'apprenant le latin, font pourtant et encore des contresens terribles :

«Et hier! Hier encore! Horresco referens, devinez, si vous l'osez, comment un autre a traduit au quatrième chant des Géorgiques ce vers :

immanis pecoris custos...

- Il me semble, répondit Michel.

- J'en rougis, jusqu'au-delà des oreilles, dit M. Richelot.

- Voyons, dites, répliqua l'oncle Huguenin ! Comment a-t-on traduit ce passage en l'an de grâce 1961 ?

- "Gardien d'une épouvantable pécore", répondit le vieux professeur en se voilant la face $^{16}$."

14 Les trois textes publiés du temps du vivant de leur auteur l'ont été chez l'éditeur parisien Hetzel. Nous citerons ces quatre romans dans les éditions modernes suivantes : Paris au XXe siècle, Paris, Hachette, 1994 ; Voyage au centre de la Terre, Paris, Gallimard, Le livre de Poche, 1998 ; Une Ville idéale : 22 illustrations originales, Amiens, Centre de documentation Jules Verne-La Maison de Jules Verne, 1999 ; Le Château des Carpathes, Paris, Gallimard, Le livre de Poche, 2011.

15 Sur l'inquiétude chez Jules Verne, voir plus particulièrement: I. MasataKa, Description de la Terre comme projet éditorial. Voyages extraordinaires de Jules Verne et système de l'éditeur Hetzel, Thèse de doctorat : Littérature et Civilisation Françaises, dir. Cl. Mouchard, Université Paris VII-Vincennes-Saint-Denis, 2007.

16 J. Verne, Paris au XXe siècle, Paris, Hachette, 1994, p. 130. 
Soit Jules Verne se trompe aussi, puisque nous avons vu que la formule s'inspire des Bucoliques et non des Géorgiques, soit il réalise volontairement cette métamorphose afin d'insister sur le caractère grotesque de la traduction opérée. Nous ne disposons cependant pas d'éléments qui nous permettraient de trancher. En outre, l'auteur cite ici le vers virgilien déjà transformé et inversé par Hugo. Il nous semble que cette transformation facilite le glissement vers une traduction plus bouffonne que ne le serait celle du vers originel - le terme de " pécore » en particulier, décalque du latin pecus, ne pouvait que faire sourire le lecteur. Dans le même roman, le héros parle ainsi de l'auteur des Contemplations : "C'est un des grands poètes du XIX ${ }^{\mathrm{e}}$ siècle, le plus grand même $[\ldots]^{17}$. » Malheureusement, Victor Hugo n'est absolument pas connu par le pauvre employé de la Librairie des Cinq parties du Monde, et pour cause, les grands classiques de la littérature n'étant plus réédités, au grand désespoir de Michel, le narrateur, qui rappelle qu'en son temps : "Notre-Dame de Paris [...] a été tiré à cinq cent mille exemplaires ${ }^{18}$. "Ce à quoi répond l'employé : " Je veux vous croire, monsieur, mais en fait de vieux auteurs réimprimés de nos jours, nous n'avons que Paul de Kock, un moraliste du siècle dernier $[\ldots]^{19}$."

Concernant la référence qui nous intéresse, nous la retrouvons, évoquée dans un contexte similaire, lorsque le romancier prononce un discours à l'Académie des sciences, belles-lettres et arts d'Amiens, le 12 décembre 1875 ("Une Ville idéale : Amiens en l'an 2000 »). Ici aussi Jules Verne parle de sa ville au futur, avec des accents ironiques ${ }^{20}$ :

"Savez-vous comment, à la version du baccalauréat, le plus fort des candidats avait traduit : Immanis pecoris custos!

- Non.

- De cette façon : "Gardien d'une immense pécore!"

- Allons donc ${ }^{21}$ !»

Dans cette nouvelle traduction, volontairement fautive, insistons sur le fait que le mot le plus grotesque ("pécore») demeure. Mais si "immense " est moins comique que " épouvantable ", il est cependant plus éloigné du sens exact du mot latin immanis.

Une fois de plus, la science et la technique ont eu raison des humanités, des études classiques qui ne sont plus au goût du jour. Le constat est amer pour cet auteur qui a

17 J. Verne, Paris au $X X^{e}$ siècle, chapitre IV " De quelques auteurs du XIX ${ }^{e}$ siècle, et de la difficulté de se les procurer », p. 58.

18 J. Verne, Paris au $X X^{e}$ siècle, p. 59.

19 J. Verne, Paris au $X X^{e}$ siècle, p. 59.

20 P. Gondolo della Riva, dans sa préface à Paris au XXe siècle, montre parfaitement comment des éléments de cet opuscule ont été remployés par Verne dans l'écriture d'Une Ville idéale, notamment la citation latine qui nous intéresse ici (J. Verne, Paris au $X X^{e}$ siècle, p. 19-20).

21 J. Verne, Une Ville idéale : 22 illustrations originales, Amiens, Centre de documentation Jules Verne, La Maison de Jules Verne, 1999, p. 48. 
obtenu son baccalauréat de philosophie et de rhétorique en 1846. Passionné de géographie, bon élève sans être excellent, Jules Verne obtient également durant sa scolarité des accessits en musique vocale, en version latine et en géographie ${ }^{22}$. Le romancier n'a pas confiance en l'avenir. Ses deux textes, périphériques aux V.E., en sont des révélateurs incontestables. Dans les deux cas, la transformation hugolienne du vers de Virgile est convoquée par le romancier pour donner à ses récits une tonalité désabusée et amère. Le vers est utilisé ici à titre de clin d'œil : le lecteur contemporain de Verne, qui a fait ses humanités, peut comprendre la double référence exploitée par le romancier.

Dans le cas des deux romans appartenant au corpus des V.E., l'incorporation de la citation virgilo-hugolienne revêt une autre dimension : elle donne forme au récit, elle lui confere un statut hautement descriptif. Elle permet d'introduire une perspective imaginaire dans un récit qui évoque un quotidien ordinaire. Commençons par le premier roman de la série des V.E. où nous trouvions cette double référence, Voyage au centre de la Terre, dans sa version de 1867, celle où l'auteur relate, en insérant des chapitres supplémentaires, la récente découverte faite par Boucher de Perthes. Cherchant l'hypothétique centre de la Terre, les héros verniens découvrent des paysages et des animaux aussi étranges qu'inattendus. Un berger monstrueux apparaît alors sous les yeux des héros ébahis :

"En effet, à moins d'un quart de mille, appuyé au tronc d'un kauris énorme, un être humain, un protée de ces contrées souterraines, un nouveau fils de Neptune, gardait cet innombrable troupeau de Mastodontes ! - Immanis pecoris custos, immanior ipse! Oui ! immanior ipse! Ce n'était plus l'être fossile dont nous avions relevé le cadavre dans l'ossuaire, c'était un géant capable de commander à ces monstres. Sa taille dépassait douze pieds. Sa tête grosse comme la tête d'un buffle, disparaissait dans les broussailles d'une chevelure inculte. On eût dit une véritable crinière, semblable à celle de l'éléphant des premiers âges. Il brandissait de la main une branche énorme, digne houlette de ce berger antédiluvien ${ }^{23}$.»

Si le glissement du berger des Bucoliques, Daphnis, au gardien de phoques terrifiant des Géorgiques, Protée, est de l'ordre de l'implicite et de l'hypothèse dans Notre-Dame de Paris, inversement le glissement opéré chez Jules Verne est parfaitement explicite. Et le berger vernien apparaît finalement aussi monstrueux que le malheureux gardien des cloches de Notre-Dame de Paris. La référence à Protée et à Neptune confirme une fois de plus que Jules Verne raccroche ce vers aux Géorgiques de Virgile, ce qui n'est pas étonnant, car le roman a été écrit peu de temps après Paris au XXe siècle. Dans son roman, Jules Verne crée finalement un alter ego de Quasimodo, évoluant dans son propre univers, souterrain ici, tout aussi fermé et inaccessible que celui dans lequel évolue le personnage hugolien. Des arches et colonnes de la cathédrale parisienne, nous

22 D. Compère, Jules Verne, parcours d'une cuvre, Amiens, Encrage, 1996, p. 11.

23 J. Verne, Voyage au centre de la Terre, chap. XXXIX, p. 318-320. 
passons alors aux arches et colonnes souterraines que les héros de Jules Verne observent et décrivent souvent par l'emploi de métaphores architecturales filées :

"La pente de cette nouvelle galerie était peu sensible, et sa section fort inégale. Parfois une succession d'arceaux se déroulait devant nos pas comme les contre-nefs d'une cathédrale gothique. Les artistes du Moyen Âge auraient pu étudier là toutes les formes de cette architecture religieuse qui a l'ogive pour générateur. Un mille plus loin, notre tête se courbait sous les cintres surbaissés du style roman, et de gros piliers engagés dans le massif pliaient sous la retombée des voûtes ${ }^{24}$."

Les deux métaphores que nous présentons ici - celle relative au monstrueux Protée vernien et celle qui décrit la pente de la galerie - procèdent d'un style hyperbolique évident. Les descriptions nous font ainsi passer d'abord de l'émerveillement à la monstruosité, puis à l'effroi et enfin aux dimensions mythiques d'une aventure littéralement hors de l'ordinaire.

La deuxième et dernière référence que nous pouvons retrouver dans les $V$.E. se situe, nous l'avons dit, dans Le Château des Carpathes, publié en 1892 : elle représente pour nous la plus significative et la plus intéressante des occurrences sur laquelle nous souhaitons concentrer notre analyse. En effet, l'emploi de la transformation hugolienne du vers de Virgile prend ici un tout autre relief. Si elle renforce l'intertextualité à laquelle recourt souvent Jules Verne, elle permet aussi à ce dernier d'achever une intratextualité qui assure au récit son déplacement à la fois dans le temps mais aussi et surtout dans l'espace.

\section{Le Château des Carpathes : intertexte et imaginaire géogra- phique dans un récit de Jules Verne}

C’est ainsi que, dès le début de son récit, Jules Verne nous présente Frik, le berger auquel la citation latine déformée se rapporte :

"Ce berger n'avait rien d'arcadien dans son accoutrement, ni de bucolique dans son attitude. Ce n'était pas Daphnis, Amyntas, Tityre, Lycidas ou Mélibée. Le Lignon ne murmurait point à ses pieds ensabotés de gros socques de bois : c'était la Sil valaque, dont les eaux fraîches et pastorales eussent été dignes de couler à travers les méandres du roman de L'Astrée.

Frik, Frik du village de Werst - ainsi se nommait ce rustique pâtour -, aussi mal tenu de sa personne que ses bêtes, bon à loger dans cette sordide crapaudière, bâtie à l'entrée du village, où ses moutons et ses porcs vivaient dans une révoltante prouacrerie -, seul 
mot, emprunté de la vieille langue, qui convienne aux pouilleuses bergeries du comitat. L'immanum pecus paissait donc sous la conduite dudit Frik - immanior ipse $[\ldots]^{25}$."

L'on remarque clairement que Jules Verne raccroche ici, enfin et justement, ce vers aux Bucoliques par des allusions explicites : " arcadien ", " bucolique "; "Daphnis, Amyntas, Tityre, Lycidas ou Mélibée ${ }^{26}$ ». Littéralement, "Le troupeau monstrueux paissait donc sous la conduite dudit Frik, - plus monstrueux lui-même ». Mais comme le montre justement Yves Touchefeu, Jules Verne se trompe en adaptant à son texte la citation latine :

"Jules Verne avait alors soixante-quatre ans. Ses souvenirs de latiniste s'estompaient.

En reprenant librement les éléments de la citation d'origine, il n'a pas oublié d'adapter les désinences du syntagme immanis pecoris, au génitif, dont il fait un nominatif. Mais il écrit immanum pecus en oubliant que l'adjectif immanis est un adjectif de la seconde classe, dont le neutre est immane, et non pas immanum ${ }^{27}$."

Or, ce n'est pas la première fois que Jules Verne commet une erreur en adaptant une citation latine. L'exemple le plus célèbre est celui de Vingt mille lieues sous les mers (1869-1870) où il écrit initialement Mobilis in mobile avant de corriger par la suite son expression par Mobilis in mobili afin de tenir compte d'une remarque faite en 1875 par le grammairien Jules Quicherat :

"Mon cher Hetzel, M. Vernes [sic] se trompe, et vous êtes dans le vrai. Les adjectifs en bilis ne sont pas de ceux qui admettent la double terminaison à l'ablatif. Ils n'en ont qu'une : celle en i. Ce n'est pas vous et moi seulement qui le disons : c'est tous ceux qui passent leur vie à faire du latin, et mon frère en premier dont j'ai eu soin à prendre l'avis quant à ce. Tout à vous, J. Quicherat ${ }^{28}$."

Jules Verne intègre également une autre référence dans son texte : L'Astrée, d'Honoré d'Urfé. Cet immense roman pastoral a été publié de 1607 à 1627, et son action principale se situe au nord de Saint-Étienne, dans la région du Forez. Le Lignon est une des rivières de cette région, et l'on remarque comment le romancier français utilise aussi cette réalité hydrographique pour établir un lien entre le récit de Virgile et celui d'Honoré d'Urfé.

La présence du berger au début de l'aventure, même anecdotique, revêt une double symbolique. Il est à la fois le cousin éloigné du berger monstrueux qui vit plusieurs kilomètres sous terre (référence interne à l'œuvre avec Voyage au centre de la Terre)

25 J. Verne, Le Château des Carpathes, chap. I, p. 8-9.

26 Daphnis, Amyntas, Tityre, Lycidas ou Mélibée appartiennent respectivement aux Bucoliques V, III, I et IX.

27 Y. Touchefeu, "Jules Verne latiniste ", p. 16.

28 BnF, NAF 16985, fo 206. Cité par J.-P. Dekiss, Jules Verne l'enchanteur, Paris, Éditions du Félin, 1999, p. 124. 
mais également un avatar de Quasimodo (référence externe à l'œuvre avec Notre-Dame de Paris). Tout cela sans oublier la source première : le berger virgilien. La métamorphose du vers opère ainsi la métamorphose du berger, invitant le lecteur à une lecture poétique à la fois de l'espace et de l'horreur. Or, il est intéressant de souligner que l'inversion que nous avons analysée procède finalement de la même inversion - ici des chronotopes romanesques - qui permet le passage du roman historique vers le roman géographique, dont les V.E. sont les représentant les plus illustres au XIX ${ }^{\mathrm{e}}$ siècle.

\section{Du roman historique au roman géographique : Les Voyages Extraordinaires de Jules Verne ou l'inversion du chronotope romanesque}

La présence de cette référence latine dans certains romans de Jules Verne participe aussi et plus largement d'une réflexion sur l'écriture du roman géographique. Pour Jean-Marie Seillan, au XIX ${ }^{\mathrm{e}}$ siècle le glissement du roman historique vers le roman géographique relève $\mathrm{du}$ passage des fictions romanesques fondées sur l'ici-autrefois aux fictions romanesques fondées sur l'ailleurs-maintenant ${ }^{29}$. "Ainsi, la concurrence faite au roman historique par le roman géographique témoigne de la rupture épistémologique autour de laquelle le $\mathrm{XIX}^{\mathrm{e}}$ siècle tout entier a pivoté ${ }^{30}$. " Or, pour Jean-Marie Seillan cette inversion chronotopique commencerait justement avec la publication des V.E. de Jules Verne.

\begin{tabular}{|c|c|c|}
\hline EsPaCE/TEMPS & ICI & Ailleurs \\
\hline MAINTENANT & $\begin{array}{l}\text { Eugénie Grandet } \\
\text { L’Assommoir }\end{array}$ & $\begin{array}{c}\text { Le Château des Carpathes } \\
\text { La Vénus noire } \\
\text { ROMAN GÉOGRAPHIQUE }\end{array}$ \\
\hline AutreFoIS & $\begin{array}{c}\text { Notre-Dame de Paris } \\
\text { Les Trois Mousquetaires } \\
\text { ROMAN HISTORIQUE }\end{array}$ & $\begin{array}{l}\text { Salammbô } \\
\text { Le Roman de la momie }\end{array}$ \\
\hline
\end{tabular}

Document 1 : L'inversion du chronotope romanesque

29 J.-M. SEILlan, "Histoire d'une révolution épistémologique au XIX siècle : la captation de l'héritage d'Alexandre Dumas par Jules Verne ", in C. Saminadayar-Perrin (dir.), Qu'est-ce qu'un événement littéraire au XIX' siècle ?, Saint-Étienne, Presses Universitaires de Saint-Étienne, 2008, p. 199-218.

30 Cf. document 1 : L'inversion du chronotope romanesque (tiré de l'article de J.-M. Seillan). Ce document, tiré de l'article de J.-M. SeIllan, a été modifié par nos soins : nous avons ajouté en italique les références aux romans historique et géographique qui nous intéressent ici, et remplacé la référence à Cinq semaines en ballon par Le Château des Carpathes, objet de notre étude. 
Jean-Marie Seillan met ainsi en évidence une inversion fondamentale qui permet de saisir comment et pourquoi Jules Verne sollicite l'imaginaire géographique dans chacun de ses romans :

" [...] le roman historique et le roman géographique reposent sur des chronotopes inversés [...] Le roman historique et le roman géographique [se] distinguent en ce que leur chronotope propre résulte de la variation d'un seul des deux paramètres. Le premier fait jouer la variable temporelle et répond à la formule ici-autrefois ; il suppose un recul chronologique et n'a pas coutume de délocaliser l'action. Le second explore les ressources de la variable spatiale et obéit au couple diagonalement opposé de l'ailleurs et du maintenant ; il propose des histoires contemporaines se déroulant dans des lieux lointains ${ }^{31}$. ”

Dans Le Château des Carpathes, la description que Jules Verne réalise de son berger repose sur un récit de type essentiellement hyperbolique. L'exagération permet au romancier de transporter le lecteur dans un autre monde, celui de l'imaginaire, de l'extraordinaire. Sa reprise de la transformation hugolienne de la citation latine lui permet de placer son récit dans une double lignée historique, à la fois ancienne et classique (Virgile) et contemporaine (Hugo). La citation latine assure un lien entre le mythe et la modernité (intertextualité/références externes), mais aussi entre les différents écrits du romancier (intratextualité/références internes). Trois imaginaires se croisent alors au travers de cette référence : l'imaginaire classique et antique de Virgile, l'imaginaire médiéval du roman hugolien et l'imaginaire contemporain du roman de Jules Verne ${ }^{32}$. Mythe, poésie et symbolique coexistent ainsi au cœur d'un seul et même roman, une coexistence cristallisée par une référence latine qui est donc loin d'être anodine pour l'auteur.

\begin{tabular}{|c|c|c|c|}
\hline Auteur & ROMAN & RÉGION & $\begin{array}{c}\text { PÉRIODE DE LA } \\
\text { NARRATION }\end{array}$ \\
\hline Virgile & Les Bucoliques & $\begin{array}{c}\text { Arcadie } \\
\text { "romanisée " }\end{array}$ & $\mathrm{I}^{\mathrm{er}}$ s. avant J.-C. \\
\hline $\begin{array}{l}\text { Honoré } \\
\text { d'Urfé }\end{array}$ & L'Astrée & Forez & $\mathrm{XVII}^{\mathrm{e}} s$. \\
\hline Victor Hugo & Notre-Dame de Paris & Paris & Moyen Âge \\
\hline Jules Verne & Voyage au centre de la Terre & Europe & 1863 \\
\hline Jules Verne & Le Château des Carpathes & Roumanie & Fin $\mathrm{XIX}^{\mathrm{e}} \mathrm{s}$. \\
\hline
\end{tabular}

Document 2: Le déplacement du récit dans le temps et dans l'espace

31 J.-M. Seillan, "Histoire d'une révolution », op. cit., p. 202.

32 Cf. document 2 : Le déplacement du récit dans le temps et dans l'espace. 
Cependant, avant d'évoquer Frik, Jules Verne cite le géographe Élisée Reclus, lorsqu'il plante le décor de la région où se déroule l'aventure :

"Ces provinces de l'extrême Europe, M. de Gérando les a décrites, Élisée Reclus les a visitées. Tous deux n'ont rien dit de la curieuse histoire sur laquelle repose ce roman. En ont-ils eu connaissance ? Peut-être, mais ils n'auront point voulu y ajouter foi. C'est regrettable, car ils l'eussent racontée, l'un avec la précision d'un analyste, l'autre avec cette poésie instinctive dont sont empreintes ses relations de voyage ${ }^{33}$."

Si la référence à Élisée Reclus permet de camper le récit dans un réel confirmé, en convoquant un argument d'autorité (Reclus et Gérando), inversement l'intégration de la citation latine relative à Frik permet de faire basculer le même récit vers l'imaginaire, le fantastique, l'extraordinaire. Cette inter/intratextualité permet donc au romancier de déplacer son récit certes dans le temps, mais surtout dans l'espace, et d'évoquer ainsi un territoire à la marge de l'Europe, la Roumanie, et plus particulièrement la Transylvanie. Le berger apparaît alors tel un intercesseur entre deux mondes, l'un réel et l'autre imaginaire. Il est par excellence la figure emblématique du vagabond, de l'errant, du marginal qui parcourt l'espace. Il n'est pas ici, il est ailleurs. Dans les V.E., plus que le temps, c'est bien l'espace qui joue un rôle fondamental dans la structuration du récit romanesque.

Jules Verne a d'ailleurs toujours reconnu écrire des romans géographiques : "Le but poursuivi par l'auteur des Voyages Extraordinaires est de dépeindre le Monde entier sous la forme du roman géographique et scientifique ${ }^{34}$ "; "Mes jeunes lecteurs savent maintenant à quels instincts, à quelles circonstances, je dois d'avoir écrit cette série de romans géographiques ${ }^{35}$ ». Enfin, une dernière citation permet de confirmer définitivement le genre dans lequel l'auteur se reconnaît :

"On m'a souvent demandé d'où m'est venue l'idée d'écrire ce qu'on peut appeler, faute d'un meilleur terme, des romans scientifiques. Eh bien, je me suis toujours attaché à l'étude de la géographie, comme d'autres pour l'histoire ou les recherches historiques. Je crois vraiment que c'est ma passion des cartes et des grands explorateurs du monde entier qui m’a amené à rédiger le premier de ma longue série de romans géographiques ${ }^{36}$. "

33 J. Verne, Le Château des Carpathes, chap. I, p. 8.

34 Lettre de Jules Verne à Louis-Jules Hetzel, 21 octobre 1888, dans Correspondance inédite de Jules Verne avec l'éditeur Louis-Jules Hetzel (1886-1914), éd O. Dumas, P. Gondolo Della Riva et V. Dehs, Genève, Slatkine - Paris, Société Jules Verne, 2004, t. I, p. 88.

35 J. Verne, Souvenirs d'enfance et de jeunesse, dans Jules Verne, Paris, L'Herne, 1974, p. 62. Les Souvenirs d'enfance et de jeunesse ont été écrits au printemps 1890 (première publication, en anglais : The Story of my Boyhood, Boston, The Youth's Companion, 1891).

36 M.A. Belloc. "Jules Verne at Home. The Strand Magazine, February, 1895 ", in D. Compère, J.-M. MARgot (éd.), Entretiens avec Jules Verne. 1873-1905, Genève, Slatkine, 1998, p. 101. 
Les V.E. de Jules Verne couronnent ainsi dans la deuxième moitié du XIX ${ }^{\mathrm{e}}$ siècle le passage du roman historique vers le roman géographique. Or, parmi les différents outils utilisés lors de l'écriture de ces romans géographiques, l'intertextualité apparaît comme un levier privilégié qui permet à l'auteur de basculer d'une géographie scientifique, où les références d'autorité (Reclus, Gérando) campent le récit dans un réel confirmé, vers une géographie plus imaginaire, fictive, portée par des références classiques, latines, virgiliennes. L'intertextualité et l'intratextualité assurent à Jules Verne l'écriture d'un imaginaire géographique qui articule dans le texte mythe et modernité.

Le Château des Carpathes est emblématique de cette démarche d'écriture. C'est en remontant ainsi aux origines de la formule analysée dans cette étude que l'on a pu mettre en évidence les liens et la filiation du romancier avec Virgile et Victor Hugo. Mais si le récit de Jules Verne fait écho à des textes et des auteurs plus anciens, il entre également en résonance avec sa propre œuvre. Un dialogue s'ouvre alors entre plusieurs récits, un dialogue cristallisé par la présence d'un berger qui apparaît pourtant comme secondaire. Le roman s'ouvre ainsi sur un espace qui déborde les seules limites du texte vernien pour communiquer avec d'autres espaces littéraires et géographiques qui alimentent l'imaginaire à la fois du romancier et du lecteur. Agissant ainsi, Jules Verne procède de cette volonté qui l'a animé toute sa vie :

"Je faisais simplement de la fiction à partir de ce qui est devenu faits ultérieurement, et mon objet n'était pas de prophétiser, mais d'apporter aux jeunes des connaissances géographiques en les enrobant d'une manière aussi intéressante que possible ${ }^{37}$."

Effectivement, si le biais de l'imaginaire permet de dire autrement l'espace géographique, il constitue aussi un formidable outil pédagogique et didactique susceptible de transmettre autrement le savoir géographique. Et l'écrivain de faire ici véritablement œuvre de géographe en convoquant une mythologie qui parcourt l'ensemble de son œuvre ${ }^{38}$.

Lionel DuPuY

Laboratoire "SET » - UMR 5603 CNRS

Université de Pau et des Pays de l'Adour

Avenue du Doyen Poplawski

Domaine universitaire - 64000 Pau

lionel.dupuy@univ-pau.fr

37 D. Compère, J.-M. Margot (éd.), Entretiens avec Jules Verne, p. 178-179.

38 Voir à ce titre S. Vierne, Jules Verne. Mythe et modernité, Paris, Puf, 1989. Par une lecture mythocritique et mythanalytique, l'auteur, spécialiste de Jules Verne, montre comment le romancier français puise fondamentalement dans le substrat mythique pour élaborer la série de ses Voyages Extraordinaires. Pour finir, nous voudrions remercier Anne-Hélène Klinger-Dollé pour ses nombreuses relectures et les suggestions qu'elle nous a faites au cours de l'écriture de cet article. 